\title{
Effect of Electrolytic Polishing on Corrosion Fatigue Strength of TiNi Shape Memory Alloy
}

\author{
Ryosuke Matsui $^{1 *}$ and Masato Okumura ${ }^{2}$ \\ ${ }^{1}$ Department of Mechanical Engineering, Aichi Institute of Technology, \\ 1247 Yachigusa, Yakusa-cho, Toyota, Aichi 470-0392, Japan \\ ${ }^{2}$ NIDEK Co., Ltd., 34-14 Maehama, Hiroishi-cho, Gamagori, Aichi 443-0038, Japan
}

(Received March 16, 2020; accepted July 8, 2020)

Keywords: shape memory alloy, TiNi alloy, corrosion, corrosion fatigue, electrolytic polishing

The corrosion fatigue strength of a TiNi shape memory alloy (SMA) is crucial for ensuring safety when the material is used as a sensor or actuator element in a severely corrosive environment, such as sea water. Furthermore, in the medical field, the corrosion fatigue strength in a blood vessel is important because it determines the product life of medical devices, such as self-expanding stents. In this study, we improved the corrosion fatigue strength of a TiNi SMA wire by electrolytic polishing. The main findings are as follows: (1) Electrolytic polishing in $25 \mathrm{wt} \%$ nitric acid and $75 \mathrm{wt} \%$ methanol at $218 \mathrm{~K}$ after mechanical polishing significantly reduces the surface roughness of the TiNi SMA wire. (2) The electrolytically polished TiNi SMA wire has a thin passive layer of $\mathrm{TiO}_{2}$ on its surface. (3) The corrosion fatigue life of the electrolytically polished TiNi SMA wire is much longer than that of a conventional TiNi SMA wire. (4) The fatigue crack on the electrolytically polished TiNi SMA wire initiates at a surface micro-imperfection.

\section{Introduction}

Shape memory materials (SMMs) are defined as substances that can revert to their original memorized shape upon an increase in temperature or other stimuli, such as magnetic field changes. ${ }^{(1,2)}$ A shape memory alloy (SMA) is a typical SMM; in particular, a TiNi-series SMA (TiNi SMA) is the most popular SMA because of its excellent recovery stress and shape-recovering properties. The material has been used as an element in temperature sensors and in many types of actuators by using the shape memory effect, which is characterized by a shape-recoverable strain with heating. ${ }^{(3)}$ To ensure the safety of these types of device, improvements in corrosion resistance and fatigue life in severely corrosive environments are extremely important.

Recently, TiNi SMAs have been used in medical devices, such as self-expanding stents. This is because of their excellent expanding force properties by means of superelasticity, ${ }^{(4)}$ which is characterized by a large shape-recoverable strain without heating. In the design of this type of stent, the corrosion fatigue life is important because this device remains in blood vessels for a long time. ${ }^{(5)}$ Furthermore, Ni ions in the TiNi SMA could dissolve in the blood through

*Corresponding author: e-mail: r_matsui@aitech.ac.jp https://doi.org/10.18494/SAM.2020.2865 
a corrosive reaction, causing allergic reactions. ${ }^{(6,7)}$ To incorporate TiNi SMAs into medical devices, several researchers have described how to improve corrosion resistance and prevent the dissolution of Ni ions. Shangqi and Xiang stated that corrosion resistance can be improved by a plasma-spraying technique involving Ti on a TiNi SMA. ${ }^{(8)}$ Liu et al. revealed that annealing can effectively enhance corrosion resistance, prevent the release of $\mathrm{Ni}$ ions, and improve cytocompatibility and antibacterial capacity. ${ }^{(9)}$ Witkowska et al. demonstrated that composite surface layers consisting of $\mathrm{C}(\mathrm{Ag})+\mathrm{TiO}_{2}$ can improve the corrosion resistance of a TiNi SMA. ${ }^{(10)}$ In terms of using TiNi SMAs in stents, researchers have investigated oxide layer damage on a TiNi SMA by several types of mechanical loading, such as bending. ${ }^{(11-13)}$ However, to date, no study has focused on the corrosion fatigue properties of SMAs in a corrosive environment.

We attempt to extend the corrosion fatigue life of a TiNi SMA wire showing the shape memory effect at room temperature using an electrolytic polishing technique to smooth the surface and possibly generate a passive layer on the exterior of the wire. The electrolytic polishing of TiNi SMAs requires the precise control of conditions compared with other commercial materials (e.g., carbon steel). Therefore, we also establish appropriate electrolytic polishing conditions, such as the electric current density, polishing time, stirring method, and temperature. Additionally, the surface quality (texture, roughness, and atomic concentration) and the fundamental deformation properties of electrolytically polished TiNi SMA wires are investigated.

\section{Materials and Methods}

\subsection{Specimen}

An as-drawn TiNi SMA wire manufactured by Furukawa Techno Material Co., Ltd., was used in this study. The wire, which had a thick oxide layer, had a composition of Ti- 49.7 at $\% \mathrm{Ni}$ and a diameter of $0.7 \mathrm{~mm}$. The preparation processes for the materials are shown in Fig. 1. The authors investigated both the corrosion resistance and corrosion fatigue life of the TiNi SMA wire prepared by a combination of mechanical and electrolytic polishing (HT-EP). Additionally, an as-heat-treated (HT) material and a mechanically polished (HT-P) material were prepared for comparison.

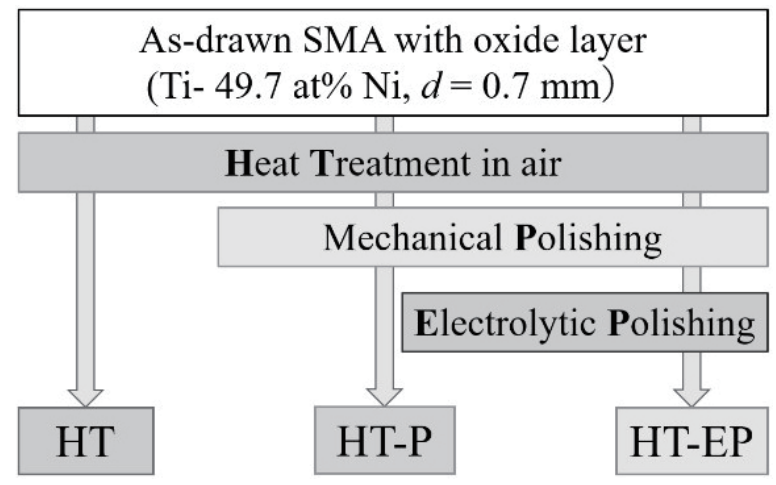

Fig. 1. Fabrication process for each material. 


\subsection{Experimental conditions}

The as-drawn TiNi SMA wire was subjected to a heat treatment in air. This is a common treatment for this material to memorize a desired shape. The treatment was conducted at $673 \mathrm{~K}$ for $3.6 \mathrm{ks}$ following furnace cooling. The material was fixed in a straight shape during the treatment in the furnace, that is, the material memorized the straight shape. The transformation temperatures of the HT material are listed in Table 1. Mechanical polishing was performed using custom-made equipment consisting of a lathe and a grinding wheel. The grinding wheel was connected to a DC motor at a rotation speed of $8700 \mathrm{rpm}$ at no load. The revolution speed of the main axis of the lathe and the feed speed of the grinding wheel were $220 \mathrm{rpm}$ and $0.18 \mathrm{~mm} / \mathrm{cycle}$, respectively. The grinding wheel numbers, which are provided in Japanese Industrial Standard (JIS R 6001), were \#240, \#600, and \#1000, and a buffing compound (corresponding to \#4000) was also used. The HT wire was evenly polished by the equipment. Electrolytic polishing was subsequently performed under the following conditions. A pure Ti plate was used as the cathode, and $25 \mathrm{wt} \%$ nitric acid and $75 \mathrm{wt} \%$ methanol at $218 \mathrm{~K}$ were used as the electrolytic solution. The HT-P wire was further polished under a current density of $237 \mathrm{~mA} / \mathrm{cm}^{2}$ for $30 \mathrm{~s}$. The corrosion resistance of all the materials was evaluated by an anodic-polarization test using an HSV-110 automatic polarization system (Hokuto Denko Corporation). The reference electrode was a saturated calomel electrode and the counter electrode was platinum. The test was conducted in a $3 \% \mathrm{NaCl}$ water solution under a scan speed of $10 \mathrm{mV} / \mathrm{min}$. Bubbling was conducted with Ar gas to decrease the amount of dissolved oxygen. The rotating bending fatigue test was performed in a $10 \% \mathrm{NaCl}$ water solution to evaluate the corrosion fatigue life of all the materials. The fatigue test was conducted under a strain amplitude $\varepsilon_{a}$ of $1-2.5 \%$ at a frequency of $100 \mathrm{cycles} / \mathrm{min}$ at $298 \mathrm{~K}$. The fatigue limit was determined as the value of $\varepsilon_{a}$ after $1 \times 10^{6}$ cycles when the material did not fracture. The fracture surface obtained by the fatigue test was observed under an optical microscope (VW-6000, Keyence Corporation) and scanning electron microscope (SEM; JSM-6700F, JEOL, Ltd.)

\section{Results and Discussion}

\subsection{Surface quality and deformation properties}

Photographs of the surface of the HT, HT-P, and HT-EP materials taken by a laser microscope (VK-X200, Keyence Corp.) and the arithmetic average roughness $R a$ along the longitudinal axis of these materials are presented in Figs. 2 and 3, respectively. The HT material has a black layer, which is the thick oxide layer already generated in the as-received material. Conversely, the surface of the HT-P material is shiny because the oxide layer generated during

Table 1

Transformation temperatures of the HT material.

\begin{tabular}{ccccccc}
\hline$M_{S}(\mathrm{~K})$ & $R_{f}(\mathrm{~K})$ & $R^{*}(\mathrm{~K})$ & $R_{S}(\mathrm{~K})$ & $A_{S}(\mathrm{~K})$ & $A^{*}(\mathrm{~K})$ & $A_{f}(\mathrm{~K})$ \\
\hline 263 & 318 & 325 & 331 & 294 & 329 & 336 \\
\hline
\end{tabular}




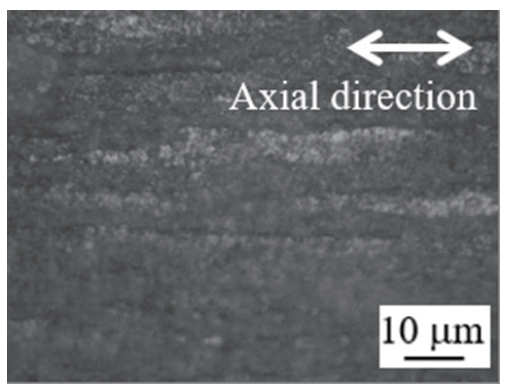

(a)

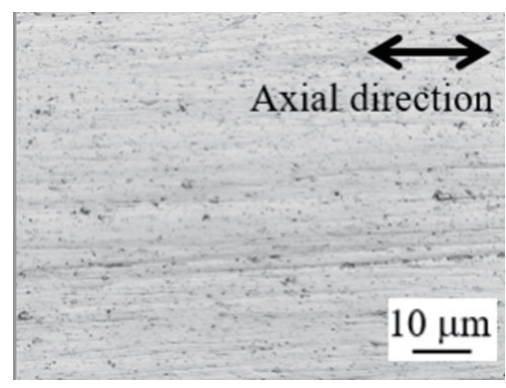

(b)

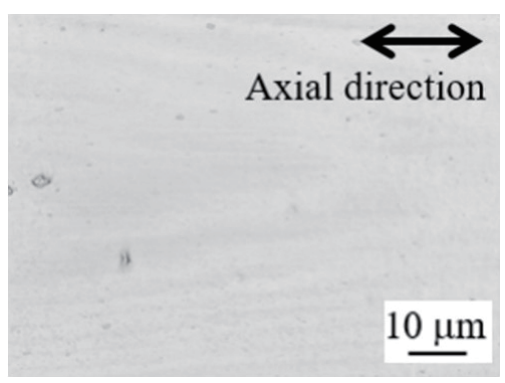

(c)

Fig. 2. Photographs of the surface of (a) HT, (b) HT-P, and (c) HT-EP materials.

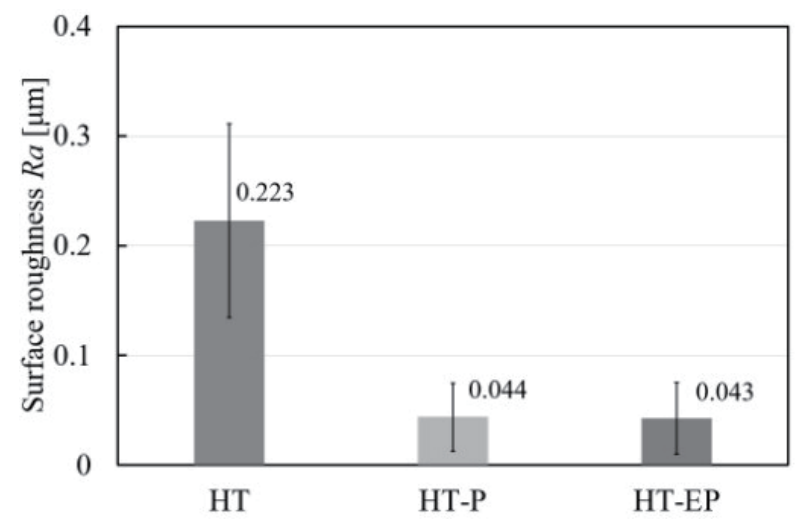

Fig. 3. $\quad R a$ for each material.

heat treatment was removed by mechanical polishing. From Fig. 3, $R a$ of the HT-P material is much lower than that of the HT material. In the case of the HT-EP material, small scratches caused by mechanical polishing were completely removed by the chemical polishing, as shown in Fig. 2(c). However, Ra of the HT-EP material is almost the same as that of the HT-P material. This suggests a useful roughness detection limit of the laser microscope.

The atomic concentration obtained from a depth analysis by X-ray photoelectron spectroscopy on the surface of the HT-EP material is presented in Fig. 4. Much carbon was detected on the outermost surface, which could be because of contamination collected during the sample fabrication process. A Ni-free layer of less than $10 \mathrm{~nm}$ thickness was detected by electrolytic polishing. From the atomic concentrations of Ti and $\mathrm{O}$, it was found that the layer consists of $\mathrm{TiO}_{2}$, which is naturally generated following electrolytic polishing.

Figure 5 shows the stress-strain curves of the HT and HT-EP materials. The tensile test was conducted at $298 \mathrm{~K}$ under a strain rate of $1 \times 10^{-4} \mathrm{~s}^{-1}$. As indicated in Fig. 5, the stress-strain curves of these materials are almost identical because their temperature histories are the same.

\subsection{Corrosion resistance}

Figure 6 shows the anodic polarization curves of each material. Although the electric potential of the HT material slightly increases in the current density region of $1 \times 10^{-4}$ to 


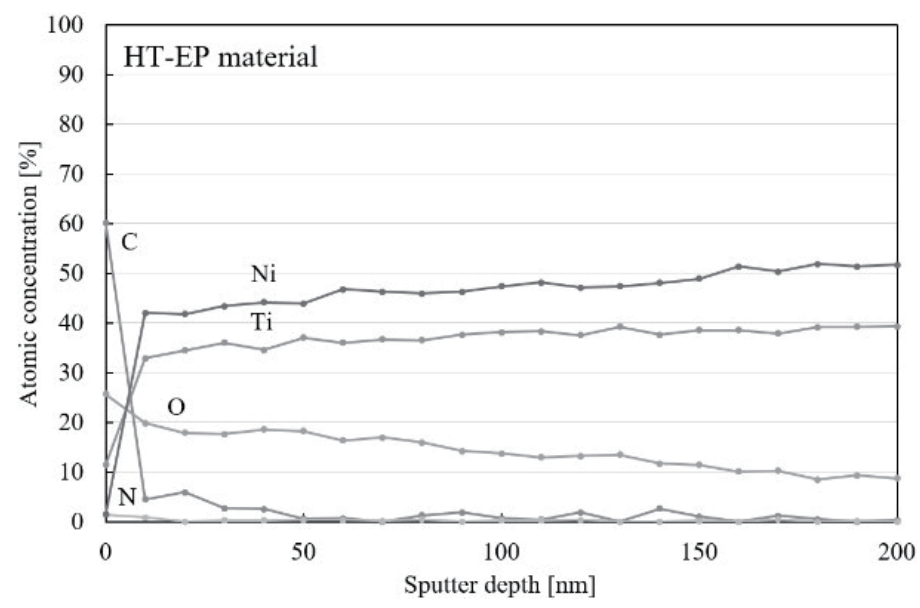

Fig. 4. Depth profile of the atomic concentrations for the HT-EP material obtained by X-ray photoelectron spectroscopy.

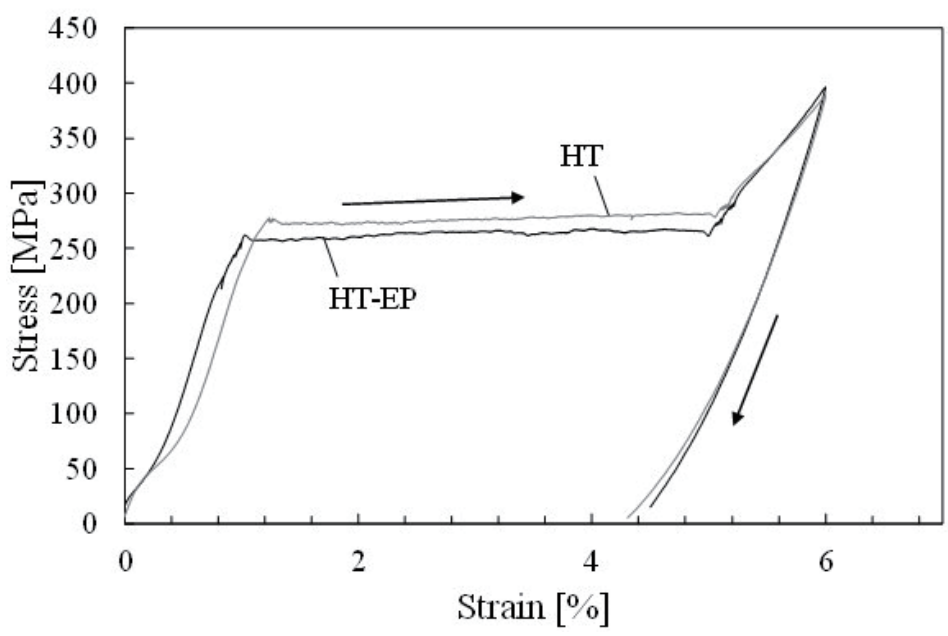

Fig. 5. Stress-strain curves for each material at 298 K.

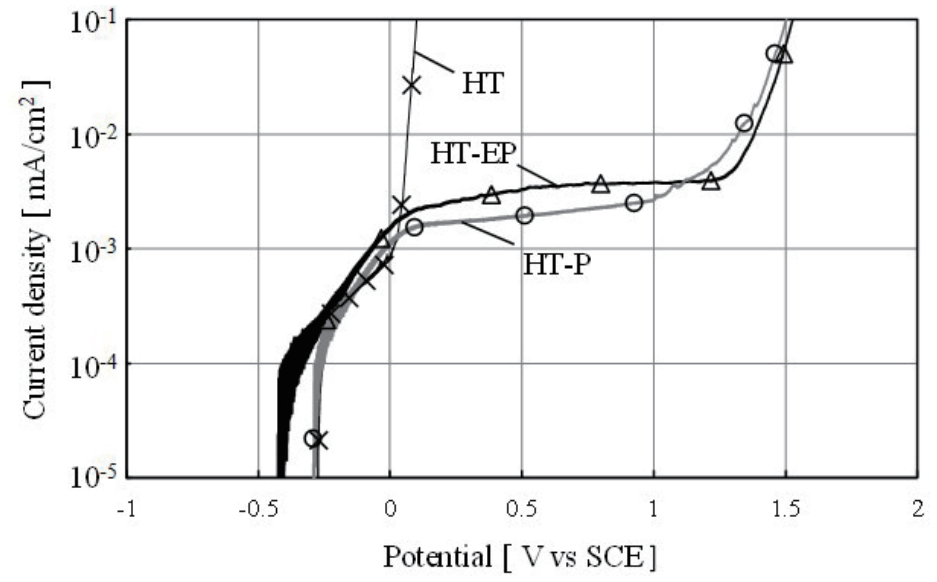

Fig. 6. Anodic polarization curves for each material. 
$1 \times 10^{-3} \mathrm{~mA} / \mathrm{cm}^{2}$, the passive regions of the HT-P and HT-EP materials are clearly observed in these curves because of the passive $\mathrm{TiO}_{2}$ layers on the surface. It is known that a $\mathrm{TiO}_{2}$ film on the surface of a TiNi SMA increases corrosion resistance, ${ }^{(14)}$ and the results agree with this tendency. Additionally, in general, for the same material, the corrosion resistance is higher with a higher surface roughness. ${ }^{(15)}$ As shown in Fig. 3, Ra of the HT material is much higher than those of the other materials; the poor surface quality of the HT material results in a deterioration in corrosion resistance. From these results, we concluded that both the existence of $\mathrm{TiO}_{2}$ and the smooth surface can greatly improve the corrosion resistance of the TiNi SMA.

\subsection{Corrosion fatigue strength}

The rotating bending fatigue lives of the materials in a $10 \% \mathrm{NaCl}$ water solution are shown in Fig. 7. There is dispersion in these results, so we plotted approximate lines obtained by the least-squares method. In the case where no fatigue fracture occurred after $1 \times 10^{6}$ cycles, $\varepsilon_{a}$ was treated as the fatigue limit and is indicated by the horizontal lines in Fig. 7. From Fig. 7, the corrosion fatigue lives of the HT-P and HT-EP materials are higher than that of the HT material because of their lower surface roughness. In particular, the fatigue life of the HT-EP material in a corrosive environment is significantly longer than those of the other materials. Because a thin passive layer exists on the surface of the HT-EP material (as shown in Fig. 4), the corrosion fatigue life of the HT-EP material is markedly extended.

To compare fatigue strength in a corrosive environment and in air, a rotating bending fatigue test in air was performed. The fatigue lives of the HT and HT-EP materials in air are also indicated in Fig. 7. In the case of the experiment in air, the experimental conditions were the same as those in the experiment in a $10 \% \mathrm{NaCl}$ water solution. As is evident in Fig. 7, the fatigue life of the $\mathrm{HT}$ material in a $10 \% \mathrm{NaCl}$ water solution is shorter than that of a material in air. This may be caused by pitting at a crack on the surface of the HT material. Conversely, in the HT-EP material, there is almost no difference between the fatigue life in a $10 \% \mathrm{NaCl}$ water solution and in air. This means that the fatigue life of the HT-EP material is independent of the environment because it has an extremely smooth surface and a thin passive layer.

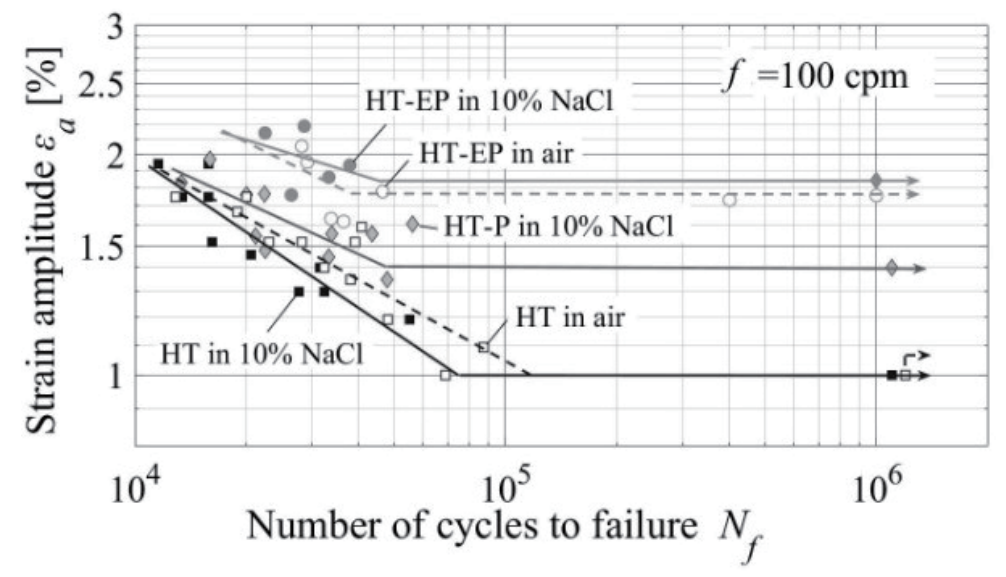

Fig. 7. Fatigue life curves for each material in a $10 \% \mathrm{NaCl}$ water solution and in air. 


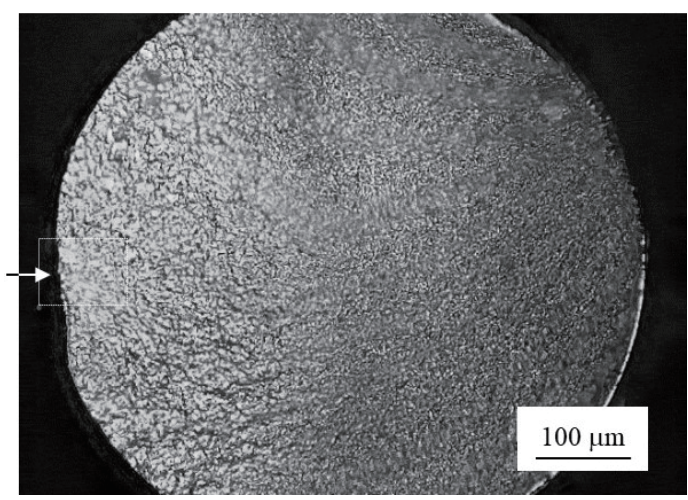

(a)

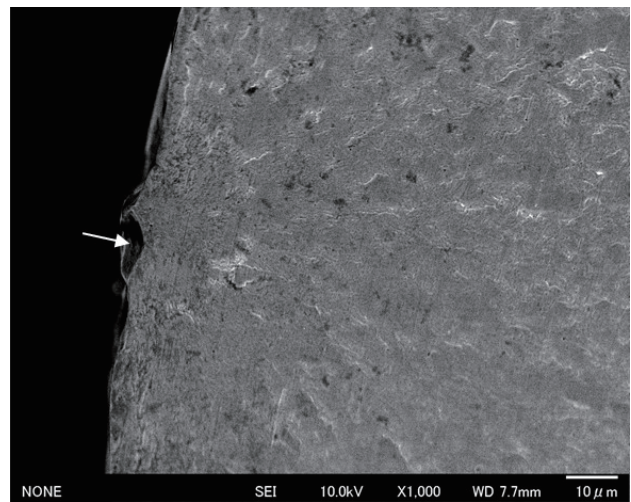

(b)

Fig. 8. (a) Fracture surface (optical microscopic image) and (b) fatigue crack initiation point (SEM image) of the HT-EP material obtained by a rotating bending fatigue test in a $10 \% \mathrm{NaCl}$ water solution $\left(\varepsilon_{a}=1.76 \%, N_{f}=26438\right)$.

Figures 8(a) and 8(b) show the fracture surfaces of the HT-EP material (obtained by an optical microscope and SEM) tested in a $10 \% \mathrm{NaCl}$ water solution. Figure $8(\mathrm{~b})$ is an enlarged view at the initiation point of the fatigue crack in the region shown by the dotted rectangle in Fig. 8(a). From Fig. 8(a), the fracture surface of the material can be classified into regions of crack progression and final fracture, as presented in another study on TiNi SMA wires. ${ }^{(16)}$ The crack nucleates at a specific point on the surface of the material and propagates toward the center in a sinuous radial pattern. As can be seen in Fig. 8(b), the crack initiates at a micro-imperfection because of stress concentration, which is indicated by the arrow. Therefore, the elimination of micro-imperfections in the electrolytic polishing process is paramount for extending the fatigue life.

\section{Conclusions}

In this study, we revealed the effects of electrolytic polishing on the corrosion resistance and corrosion fatigue strength of a TiNi SMA wire. The main findings are as follows:

(1) Electrolytic polishing in $25 \mathrm{wt} \%$ nitric acid and $75 \mathrm{wt} \%$ methanol at $218 \mathrm{~K}$ after mechanical polishing significantly reduced the surface roughness of the TiNi SMA wire.

(2) The electrolytically polished TiNi SMA wire had a thin passive layer of $\mathrm{TiO}_{2}$ on its surface.

(3) The corrosion fatigue life of the electrolytically polished TiNi SMA wire was significantly longer than that of a conventional TiNi SMA wire.

(4) The fatigue crack of the electrolytically polished TiNi SMA wire initiated at a microimperfection on the surface and propagated toward the center in a sinuous radial pattern.

\section{Acknowledgments}

This work was supported by JSPS KAKENHI Grant Number 19K05066 and the Hibi Science Foundation. 


\section{References}

1 K. Otsuka and X. Ren: Prog. Mater. Sci. 50 (2005) 511. https://doi.org/10.1016/j.pmatsci.2004.10.001

2 H. Miki, K. Tsuchiya, M. Ohtsuka, M. Gueltig, M. Kohl, and T. Takagi: Advances in Shape Memory Alloy, Q. Sun, R. Matsui, K. Takeda, and E. A. Pieczyska, Eds. (Springer International Publishing, Cham, 2017) Chap. 11. https://doi.org/10.1007/978-3-319-53306-3_11

3 J. M. Jani, M. Leary, A. Subic, and M. A. Gibson: Mater. Des. 56 (2014) 1078. https://doi.org/10.1016/ j.matdes.2013.11.084

4 H. Tobushi, R. Matsui, K. Takeda, and E. A. Pieczyska: Shape Memory Effect and Superelasticity: Mechanical Properties of Shape Memory Materials (Nova Science Publishers, New York, 2013) Chap. 1. https:// novapublishers.com/shop/mechanical-properties-of-shape-memory-materials/

5 D. Stoeckel, A. Pelton, and T. Duerig: Eur. Radiol. 14 (2004) 292. https://doi.org/10.1007/s00330-003-2022-5

6 C. L. Chu, R. M. Wang, T. Hu, L. H. Yin, Y. P. Pu, P. H. Lin, S. L. Wu, C. Y. Chung, K. W. K. Yeung, and P. K. Chu: Mater. Sci. Eng. C 28 (2008) 1430. https://doi.org/10.1016/j.msec.2008.03.009

7 M. Morita, T. Hashimoto, K. Yamauchi, Y. Suto, T. Homma, and Y. Kimura: Mater. Trans. 48 (2007) 352. https://doi.org/10.2320/matertrans.48.352

8 S. Shangqi and L. Xiang: Chem. Eng. Trans. 59 (2017) 769. https://doi.org/10.3303/CET1759129

9 Y. Liu, R. Hang, Y. Zhao, L. Bai, Y. Sun, X. Yao, H. Jia, B. Tang, and R. Hang: Surf. Coat. Technol. 352 (2018) 175. https://doi.org/10.1016/j.surfcoat.2018.08.016

10 J. Witkowska, A. Sowinska, E. Czarnowska, T. Plocinski, B. Rajchel, M. Tarnowski, and T. Wierzchon: J. Mater. Sci. Mater. Med. 29 (2017) 110. https://doi.org/10.1007/s10856-018-6118-5

11 S. Shabalovskaya, G. Rondelli, and M. Rettenmayr: J. Mater. Eng. Perform. 18 (2009) 470. https://doi. org/10.1007/s11665-009-9498-3

12 S. Shabalovskaya, G. Rondelli, A. Undisz, J. Anderegg, T. Burleigh, and M. Tettenmayr: Biomaterials 30 (2009) 3662. https://doi.org/10.1016/j.biomaterials.2009.03.034

13 C. Heßing, J. Frenzel, M. Poul, and S. A. Shabalovskaya: J. Mater. Sci. Eng. A 486 (2008) 461. https://doi. org/10.1016/j.msea.2007.09.029

14 Y. Kimura: WIT Trans. Eng. Sci. 71 (2011) 149. https://doi.org/10.2495/978-1-78466-249-3/009

15 E. Arslan, Y. Demirci, and A. Alsaran: J. Mater. Eng. Perform. 19 (2010) 428. https://doi.org/10.1007/s11665009-9504-9

16 R. Matsui, H. Tobushi, Y. Furuichi, and H. Horikawa: J. Eng. Mater. Technol. 126 (2004) 384. https://doi. org/10.1115/1.1789952 